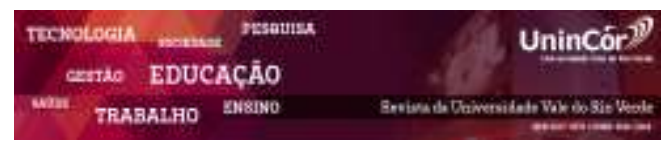

Revista da Universidade Vale do Rio Verde ISSN: 1517-0276 / EISSN: 2236-5362 Vol. 16 | n. 1 | Ano 2018

Marllon Thadeu da Costa Ramos Universidade do Estado do Pará marllonthadeu@gmail.com

Luan Oliveira da Silva Universidade do Estado do Pará luanosilva_17@hotmail.com

Thalita da Luz Costa Universidade do Estado do Pará thalitadaluzcosta@gmail.com

Lorena de Almeida Costa Universidade do Estado do Pará lorenadeac.2013@gmail.com

Renata Amanajás de Melo Universidade do Estado do Pará. rennamelo@uol.com.br

\section{A INFLUÊNCIA DA REALIDADE VIRTUAL NO EQUILÍBRIO E NA QUALIDADE DE VIDA DOS PORTADORES DE DOENÇA DE PARKINSON}

\section{RESUMO}

A Doença de Parkinson (DP) é uma patologia de caráter debilitante e com grande acometimento motor progressivo. Essa afecção neurológica acomete principalmente a população idosa, apresenta déficit motor global e outras alterações não motoras que ocasionam um déficit de equilíbrio e na qualidade de vida. Como objetivo de e retardar a evolução desses sinais e sintomas, a fisioterapia promove melhora da capacidade funcional através de recursos que otimizam a função motora desses pacientes. Um desses recursos é a realidade virtual que consiste na reprodução de ações motoras através de sensores de movimentos, muito utilizado em reabilitação de disfunções motoras e cognitiva. Objetivo desse estudo foi avaliar a influência da realidade virtual no equilíbrio e na qualidade de vida dos portadores de DP. Foram incluídos 6 indivíduos com DP com média de idade de $66 \pm 9,5$ anos, os quais submeteram-se análise do equilíbrio, pelo teste de tinetti, e análise da qualidade de vida, pelo questionário PDQL. Avaliação do equilíbrio e da qualidade de vida foi realizada antes e após as 15 seções de terapia com realidade virtual. Os dados coletados foram analisados através do teste $\mathrm{t}$ de student, onde foram encontradas variáveis estatisticamente significantes no teste te tinetti ( $\mathrm{p}<0,0013$ no equilíbrio estático e $\mathrm{p}<0,0013$ no equilíbrio dinâmico) e no questionário PDQL ( $\mathrm{p}<0,006)$. Através dos dados obtidos concluímos que houve influência positiva no equilíbrio e na qualidade de vida dos portadores de DP.

Palavras-chave: Doença de Parkinson. Terapia de Exposição a Realidade Virtual. Jogos de Vídeo Game.

\section{THE INFLUENCE OF VIRTUAL REALITY IN BALANCE AND QUALITY OF LIFE OF PATIENTS WITH PARKINSON'S DISEASE}

\begin{abstract}
Parkinson's disease (PD) is a debilitating condition with great character and progressive motor impairment. This neurological disease mainly affects the elderly population presents global motor deficit and other non- motor abnormalities that cause a deficit of balance and quality of life. How goal and slow the progress of these signs and symptoms, physical therapy promotes improved functional capacity through capabilities that optimize motor function in these patients. One of these features is the virtual reality that is the reproduction of motor actions using motion sensors, widely used in rehabilitation of motor and cognitive dysfunctions. Objective of this study was to evaluate the influence of virtual reality in balance and quality of life of patients
\end{abstract}


with PD. 6 PD patients with mean age of $66 \pm 9.5$ years, who underwent equilibrium analysis, by Tinetti test, and analysis of quality of life, questionnaire PDQL were included. Assessment of balance and quality of life was performed before and after 15 therapy sessions with virtual reality. The collected data were analyzed using the Student's t test, where statistically significant variables were found in thee Tinetti test $(\mathrm{p}<0.0013$ in static equilibrium and $\mathrm{p}<0.0013$ in dynamic equilibrium ) and PDQL questionnaire $(\mathrm{p}<0.006)$. Through the data we conclude that there was a positive influence on balance and quality of life of patients with PD.

Keywords: Parkinson Diease. Virtual Reality Exposure. Video Game.

\section{Recebido em: 17/11/2017 - Aprovado em: 18/01/2018 - Disponibilizado em: 15/07/2018}

\section{INTRODUÇÃO}

A DP foi descrita a primeira vez em 1817 por James Parkinson, no ensaio intitulado de "An Essay On The Shaking Palsy" (Ensaio Clínico Sobre a Paralisia Agitante), sendo atualmente descrita como uma patologia crônicodegenerativa do sistema nervoso central que acomete neurônios dopaminérgicos da substância negra, ocasionando redução de dopamina na vianigroestrital (MELO; BOTELHO, 2010).

Ela é caracterizada por uma tétrade clinica específica, formada por tremor de repouso, bradcinesia, rigidez muscular e instabilidade postural. Sua etiologia especifica é incerta, porém alguns autores consideram influência de fatores hereditários, tóxicos, genéticos e ambientais (MASCHARENAS; SOUZA, 2010; ROWLAND, 2003).

De acordo com Melo e Botelho (2010) a DP é umas das patologias neurodegenerativas que mais acometem os idosos, apresentando uma incidência de 1 a cada 1000 acometimentos em indivíduos acima de 65 anos e 1 a cada 100 indivíduos acima de 75 anos, sendo a população masculina mais afetada na proporção de 2:1

Associado as manifestações principais da patologia podemos observar outros sinais motores, como o déficit de planejamento e aprendizado motor, alterações da marcha e o fenômeno do congelamento. Além dos sinais motores, a DP apresenta sinais não motores, como: alterações neurocomportamentais, como demência, depressão e tenência ao isolamento (MELO; BOTELHO, 2010; O'SULLIVAN, 2010; ROWLAND, 2003).

Dentre todos os sintomas supracitados, o déficit de equilíbrio, tanto estático e quanto dinâmico, é a alteração mais frequente nos portadores de DP (CHRISTOFOLETI, et al, 2010).

Segundo Christofoletti et al (2010), o tratamento deve ser individualizado e composto de uma equipe multidisciplinar, pois cada paciente possui um quadro singular de sinais, sintomas, resposta medicamentosa, necessidades, ocupacionais e psicossociais que devem ser levadas em consideração. 
Dentre esses profissionais, o fisioterapeuta tem um papel fundamental no controle dos sintomas da DP, onde através de recursos e técnicas como PNF, hidroterapia, mecanoterapia e cinesioterapia, consegue retardar a evolução dos sintomas e melhorar a mobilidade, força muscular, equilíbrio e com isso proporcionar uma melhor qualidade de vida aos portadores dessa patologia (SANTOS et al, 2010).

Com o a evolução da informática novas formas de reabilitação estão surgindo objetivando melhorar a qualidade do tratamento. A realidade virtual é uns desses recursos que vem sendo utilizado no tratamento de portadores de disfunções cognitiva e motora. Ela consiste na simulação de um ambiente real, onde são reproduzidas ações motoras através de sensores de movimentos, havendo uma interação entre o indivíduo e a máquina (SCHIAVIANATO et al, 2010).

Os benefícios associados a esse tipo de reabilitação são: correção de postura e equilíbrio, melhoria da locomoção e funcionalidade dos membros superiores e inferiores, além de promover motivação do paciente SCHIAVIANATO et al, 2010; CAETANO; MALAGUTTI, 2012; BARCALA et al, 2011)

Segundo Caetano e Malagutti (2012) o incremento desse recurso pode ser um facilitador da realização dos movimentos durante a reabilitação dos pacientes com DP. Ainda de acordo com esses autores, o fato de portadores de DP não apresentarem déficit cognitivos, perceptuais e sensoriais graves, principalmente durante as fases iniciais favorecem aplicabilidade do recurso.
Diante das incapacidades que DP promove e a fim de se obter mais conhecimentos nessa área de atuação da fisioterapia, esse estudo tem como objetivo avaliar a influência que a realidade virtual promove sobre no equilíbrio e na qualidade de vida dos portadores dessa patologia.

\section{MÉTODOS}

Esta pesquisa A pesquisa foi realizada após o aceite do orientador, o aceite do local da pesquisa, a submissão e aprovação pelo Comitê de Ética em Pesquisa da Universidade do Estado do Pará (UEPA) do Centro de Ciências Biológicas e da Saúde (CCBS) - Campus II, e a assinatura do Termo de Consentimento Livre e Esclarecido (TCLE) pelos voluntários da pesquisa. Os indivíduos foram estudados segundo os preceitos da Declaração de Helsinque e do Código de Nuremberg, sendo respeitadas as normas de pesquisas envolvendo seres humanos (Res. CNS 466/12) do Conselho Nacional de Saúde.

Este estudo experimental e longitudinal foi realizado com pacientes que se encontravam na lista de espera da Unidade de Ensino e Assistência a fisioterapia e Terapia Ocupacional (UEAFTO) da Universidade do Estado do Pará. Foram incluídos na pesquisa indivíduos portadores de DP de ambos os sexos, na faixa etária entre 45 a 85 anos, com escore de entre 0 a 3 na escala de Hoehn e Yar modificada (EHY), sem uso de dispositivo para marcha, cadastrados na UEAFTO e que autorizaram a pesquisa, assinando o Termo de Consentimento Livre e Esclarecido (TCLE). Sendo excluídos os indivíduos que possuíssem Mini-Mental com score menor de $\leq$ 
22, score superior a 3 na HY, convulsões, hipertensão descompensada, cardiopatia ou qualquer outra patologia associada a DP.

A partir da lista de espera da UEAFTO, foram selecionados 21 pacientes para compor amostra, destes, 15 foram excluídos, 4 por recusarem participar da pesquisa, 2 por idade acima de 85 anos, 3 por apresentarem escore da EHY acima de 3, 6 por terem respondido ao contato.

Após o contato via telefone, foi agendado um encontro com cada paciente, em horários diferentes, onde foram esclarecidos os objetivos da pesquisa, o qual consistiu no tratamento com realidade virtual além dos riscos e benefícios que a pesquisa pudesse oferecer. Aqueles que concordarem em participar do estudo foi apresentado o TCLE.

Após assinatura do TCLE, os pacientes foram submetidos ao Mini Mental para análise da cognição e à EHY para verificar a evolução da DP.

Os que obtiveram os escores do Mini Mental e da EHY dentro dos critérios de inclusão da pesquisa, foram submetidos ao questionário PDQL (Parkinson Disease Quality of Life) e teste de Tinetti para avaliar o equilíbrio estático e dinâmico. Concluído a etapa inicial de avalição, os pacientes que atenderem aos critérios de inclusão iniciaram o tratamento com realidade virtual.

No protocolo com realidade virtual foi utilizado o jogo Sports Champions do console de videogame PlayStation ${ }^{\circledR} 3$ da Sony Computer Entertainment ${ }^{\circledR}$, associado com a PlayStation ${ }^{\circledR}$ Eye e o controle de movimento PlayStation ${ }^{\circledR}$ Move. O paciente foi orientado sobre o funcionamento do aparelho e do jogo antes do início do tratamento. A modalidade escolhida do jogo foi o voleibol, onde o paciente reproduzia movimentos de básicos do jogo, tais como:

- Manchete: Extensão de cotovelo e $90^{\circ}$ de flexão de ombro.

- Bloqueio: $180^{\circ}$ de Flexão de ombro com extensão de cotovelo.

- Corte e Saque: Flexão de ombro seguida de extensão de ombro.

Dessa forma, foi montado um circuito de exercícios, que consistia de uma série de saque, de bloqueio e de manchete, sendo executado 8 repetições para cada exercício. O circuito era realizado 2 vezes, com 1(um) minuto de intervalo entre cada modalidade, bem como entre a primeira e segunda circuito. Antes da primeira intervenção foi realizada uma sessão de treinamento com equipamento e o jogo utilizado.

A tela de projeção da imagem foi posicionada a 2 metros de distância do paciente, conforme indicação do manual do aparelho. O protocolo foi aplicado 3 vezes por semana, em dias alternados, durante 5 semanas consecutivas, totalizando 15 sessões com duração aproximada de 20 minutos.

Ao Final do tratamento foi reaplicada a escala de Tinetti, com objetivo de analisar a evolução do equilíbrio dinâmico e PDQL dos indivíduos tratados.

Após a coleta de dados foi elaborado uma planilha eletrônica, para armazenamento dos dados, no software Microsoft Excel® 2010, na qual cada linha corresponde a um caso e cada coluna a uma variável. Para representação descritiva dos dados foram elaboradas tabelas, utilizando o Software Microsoft Word®, 
representando as médias e desvio-padrão das distribuições das variáveis numéricas. A análise dos dados foi processada utilizando-se o programa SPSS Statistics 17.0. Para comparação das médias das variáveis numéricas de equilíbrio

\section{RESULTADOS E DISCUSSÃO}

Foram incluídos na pesquisa 6 pacientes, sendo 3 do sexo masculino e 3 do sexo feminino com média de idade de $66 \pm 10,4$ anos. O quadro1 apresenta a descrição de amostra, em quanto o quadro 2 apresenta a evolução do equilíbrio e da qualidade de vida pelos escores do teste de tinetti e do questionário PDQL respectivamente.

Quadro 1 - Descrição da Amostra

\begin{tabular}{llll}
\hline & Sexo & Idade & EHY \\
\hline $\mathrm{P}_{1}$ & $\mathrm{~F}$ & 50 & 1 \\
$\mathrm{P}_{2}$ & $\mathrm{~F}$ & 81 & 2,5 \\
$\mathrm{P}_{3}$ & $\mathrm{M}$ & 66 & 3 \\
$\mathrm{P}_{4}$ & 70 & $\mathrm{M}$ & 2 \\
$\mathrm{P}_{5}$ & 69 & $\mathrm{M}$ & 2 \\
$\mathrm{P}_{6}$ & 60 & $\mathrm{~F}$ & 1 \\
\hline
\end{tabular}

Fonte: Pesquisa de Campo

Quadro 2 - Evolução do Equilíbrio e da Qualidade de Vida em do paciente com DP

\begin{tabular}{lllll}
\hline & Tinetti & PDQL & & \\
\hline & Inicial & Final & Inicial & Final \\
P1 & 18 & 22 & 95 & 102 \\
P2 & 12 & 14 & 91 & 98 \\
P3 & 12 & 15 & 60 & 78 \\
P4 & 12 & 16 & 109 & 130 \\
P5 & 16 & 18 & 94 & 105 \\
P6 & 8 & 10 & 94 & 105 \\
\hline
\end{tabular}

Fonte: Pesquisa de Campo

A tabela 2 apresenta a descrição da média e desvio-padrão das variáveis do teste de tinetti, no início e final do tratamento do com realidade virtual, havendo melhora significativa tanto no estático, equilíbrio dinâmico, escore total e PDQL antes e depois do tratamento utilizou-se o teste $\mathrm{t}$ de Student para amostras pareadas, considerando-se nível de significância de 5\% (pvalor $<0,05)$.

equilíbrio estático quanto o dinâmico, pois foi obtivo um p-valor $<0,05$.

Tabela 1 - Média e desvio-padrão das variáveis do Teste de tinetti, no início e no final do tratamento dos pacientes com DP da UEAFTO.

\begin{tabular}{lccccc}
\hline \multicolumn{1}{c}{ Variável } & \multicolumn{2}{c}{ Inicial } & \multicolumn{3}{c}{ Final } \\
\hline & Média & $\begin{array}{l}\text { Desvio- } \\
\text { padrão }\end{array}$ & Média & $\begin{array}{l}\text { Desvio- } \\
\text { padrão }\end{array}$ & $\begin{array}{l}\text { p- } \\
\text { valor }\end{array}$ \\
\hline $\begin{array}{l}\text { Equilíbrio } \\
\text { estático }\end{array}$ & 9,5 & 2,3 & 3,5 & 1,5 & 0,0013 \\
$\begin{array}{l}\text { Equilíbrio } \\
\text { dinâmico }\end{array}$ & 11,1 & 2,4 & 4,8 & 2,1 & 0,0013 \\
$\begin{array}{l}\text { Escore } \\
\text { total }\end{array}$ & 13 & 3,5 & 15,8 & 4 & 0,0008 \\
& & & & &
\end{tabular}

Fonte: Pesquisa de campo

Assim com o equilíbrio, obteve-se uma melhora significativa na qualidade após a aplicação da realidade virtual, com base na análise do PDQL aplicado antes e após o tratamento, como mostra a tabela 2.

Tabela 2 - Média e desvio-padrão da variável PDQL, no início e no final do tratamento dos pacientes com DP da UEAFTO

\begin{tabular}{llllll}
\hline & Inicial & \multicolumn{3}{c}{ Final } \\
\cline { 2 - 6 } Variável & Média & $\begin{array}{l}\text { Desvio- } \\
\text { padrão }\end{array}$ & Média & $\begin{array}{l}\text { Desvio- } \\
\text { padrão }\end{array}$ & p-valor \\
\hline $\begin{array}{l}\text { Escore } \\
\text { PDQL }\end{array}$ & 93,5 & 18,5 & 104,6 & 18,2 & 0,006 \\
\hline
\end{tabular}

Fonte: Pesquisa de campo

A realidade virtual promove um feedback visual extrínseco quando, através das mudanças de movimentos gerado pelo ambiente virtual, 
estimula o indivíduo a criar estratégias neuromusculares que melhorem o equilíbrio corpóreo, além de sua qualidade de vida (ZEIGELBOIM et al, 2013; JUNIOR; SILVA 2012).

Nesse presente estudo foi possível observar que a terapia com realidade virtual apresentou uma melhora significativa no equilíbrio e na qualidade de vida de pacientes portadores de DP, corroborando com estudos de Loureiro et al. (2012) que recrutaram seis voluntários com média de idade de $65 \pm 13$ anos, obtendo resultados significativos após 12 aplicações de terapia com realidade virtual.

O incremento da simulação de movimentos no programa de reabilitação de portadores de déficit neuromotor vem crescendo e mostrando-se efetivo na recuperação de atraso neurológico. Estudos realizados por de Escuilier et al. (2012) demonstraram que houve uma melhora significativa no escore do teste de tinetti, tanto no equilíbrio estático quanto no dinâmico, após o protocolo de realidade virtual, aumentando, em média o escore em 4 pontos, após 18 semanas de tratamento. Resultados esses que se assemelham com os da presente pesquisa onde foi possível elevar o escore total, em média, 2,8. Ratificando a melhora no equilíbrio dos pacientes do estudo.

A melhora do equilíbrio dinâmico, que foi alcançada no presente e no estudo dos autores anteriores vai de encontra o estudo de Gil-Gómez et al. (2011) que só obtiveram resposta significativa ao equilíbrio estático. Segundo o mesmo autor isso pode estar relacionado ao console utilizado na pesquisa, onde os movimentos devem ser executados em cima de uma plataforma. Sendo esse um dos motivos principais de ser sido escolhido o PlayStation $® 3$, pois permite o deslocamento do usuário.

Este estudo também obteve uma melhora significativa na qualidade de vida dos portadores de DP, a partir da análise dos escores inicial e final do PDQL. Segundo estudos De Boer et al. (1996) quanto maior o escore no PDQL, melhor é a qualidade de vida do indivíduo.

Segundo Loureiro et al. (2012) o incremento de um ambiente virtual pode, além de proporcionar a melhora da capacidade funcional, facilitar a prática do lazer que é indispensável para uma boa qualidade de vida. Justificando assim a melhora na qualidade de vida dos pacientes desse presente estudo, pois foi utilizado um simulador de jogo de vôlei para a terapia com realidade virtual.

Apesar de não ser um dos objetivos da pesquisa, mas houve relato de todos os pacientes numa melhora da mobilidade articular dos membros superiores e do divertimento durante o tratamento. O que corrobora com o estudo de Loureiro et al. (2012) que avaliou a viabilidade de da aplicação da realidade virtual no tratamento da DP.

Outro dado importante a ser exposto foi que dentre os 6 indivíduos que participaram do presente estudo, apenas um apresentou dificuldade com o manuseio do aparelho e com a execução dos exercícios no início do tratamento, o que pode ser revertido com o tempo. Dado esse que corrobora com os estudos de Pompeu et al. (2012), no qual avaliaram o desempenho dos portadores de DP em 10 jogos, não apresentando déficit de aprendizagem em 7. Os mesmos autores ressaltam a importância da seleção adequado dos 
jogos para se obter um desempenho satisfatório durante o programa de reabilitação.

\section{CONCLUSÃO}

A partir da análise dos dados obtidos foi possível constar uma influência positiva no equilíbrio estático e dinâmico e na qualidade de vida dos portadores de doenças de DP.

Vale ressaltar que apesar de fácil aplicabilidade da terapia, se faz necessário a presença de um profissional capacitado que tenha conhecimentos dos dispositivos a serem utilizados e da patologia a que será tratada, pois para cada indivíduo existe uma particularidade e um objetivo a ser alcançado, sendo o terapeuta responsável por escolher mais adequado.

\section{REFERÊNCIAS}

BARCALA, B. et al. Analise do Equilíbrio em Paciente Hemiparéticos Após o Treino com o Programa Wii Fit. Fisioter. Mov. v. 24, n. 2, p. 337-343, 2011

CAETANO, KC; MALAGUTTI. W. Informática em Saúde: Uma Perspectiva Multiprofissional dos Usos e Possibilidade. São Paulo: Yends, 2012.

CHRISTOFOLETTI, G; et al. Eficácia do Tratamento Fisioterapêutico no Equilíbrio Estático e Dinâmico de Pacientes com Doença de Pakinson. Fisioter Pesq, v. 17, n. 3, p.259-263, 2010.

DE BOER, A G E M. et al. Quality of life in patients with Parkinson's disease: development of a questionnaire. J Neurol Neurosurg Psychiatry v. 61, n.1, p. 70-74, 1996.

ESCUILIER, J-C. et al. Home-based Balance Training Programm e Using Wii Fit with Balance Board for Parkinson's Disease: A Pilot Study. J Rehabil Med v.44, n. 1, p.144-150, 2012

GIL-GÓMEZ, JA. Et al. Effectiveness of a Wii balance board-based system (eBaViR) for balance rehabilitation: a pilot randomized clinical trial in patients with acquired brain injury. Journal of NeuroEngineering and Rehabilitation,v.8, n.3, 2011.

JUNIOR, RSM; Silva, EB. Efetividade da reabilitação virtual no equilíbrio corporal e habilidades motoras de indivíduos com défi cit neuromotor: uma revisão sistemática. Rev Bras Ativ Fis e Saúde. v. 17, n.3, p.224-230, 2012

LOUREIRO, APC. et al. Feasibility of virtual therapy in rehabilitation of Parkinson's disease patients: pilot study Fisioter Mov. v.25, n. 3, p. 659-66, 2012.

MASCARENHAS, CHM; SOUZA, MP. Avaliação Funcional de Indivíduos Portadores da Doença de Parkinson. Arq Ciênc Saúde, v.17, n. 4, p. 179-184, 2010

MELO, M.P.B.; BOTELHO, A.C.G. Correlação das Escalas de Avaliação Utilizadas na Doença de Parkinson com Aplicabilidade na Fisioterapia. Fisioter. Mov, v. 23, n.1, p.121-127, 2010.

MELO-SOUZA; SE. Tratamento das Doenças Neurológicas. 2 ed. Rio de Janeiro: Guanabara Koogan, 2008.

O’SULLIVAN, SB; schmitz, TJ. Fisioterapia: avaliação e tratamento. 5. ed. São Paulo: Manole, 2010.

POMPEU, JE. et al. Motor Learning, Retention and Transfer After Virtual-Reality-Based Training in Parkinson's Disease--Effect of Motor and Cognitive Demands of Games: A Longitudinal, Controlled Clinical Study. Physiotherapy, v. 98, n.3, p. 217-23, 2012.

ROWLAND, L P. Merritt tratado de neurologia. 10. ed. Rio de Janeiro: Guanabara Koogan, 2002. Santos, VV. et al. Fisioterapia na Doença de Parkinson: Uma Breve Revisão. Rev Bras Neurol, v. 46, n. 2, p. 17-25, 2010

SCHIAVINATO, AM et al. Influência do Wii Fit no Equilíbrio de Paciente com Disfunção Cerebelar: Estado de Caso. J Health Sci Inst. v. 28, n.1, p.50-52.

Zeigelboim BS. et al. Reabilitação vestibular com realidade virtual na ataxia espinocerebelar: Relato de Caso. ACR, v. 18, n. 2, p. 143-7, 2013 


\begin{tabular}{l}
\hline Marllon Thadeu da Costa Ramos \\
Pós-graduado em Acupuntura Tradicional e \\
Integrativa pela Faculdade Inspirar.
\end{tabular}

\section{Luan Oliveira da Silva}

Pós-graduado em Fisioterapia Hospitalar pelo Centro

Universitário do Estado do Pará.

\begin{tabular}{l}
\hline Thalita da Luz Costa \\
Especialista em Fisioterapia Oncológica pela \\
Universidade Federal do Pará.
\end{tabular}

Universidade Federal do Pará.

\begin{tabular}{l}
\hline Lorena de Almeida Costa \\
Especialista em Fisioterapia na Urgência e \\
Emergência no Trauma pela Universidade do Estado \\
do Pará, Pós-graduada em Fisioterapia na Unidade de \\
Terapia Intensiva pela Faculdade Inspirar. \\
\hline
\end{tabular}

Renata Amanajás de Melo
Mestre, Mestre em Ciências da Motricidade Humana
pela Universidade do Estado do Pará, Chefe do
DCMH da UEPA, Coordenadora de estágio da
UNAMA

